\title{
PERFIL SÓCIO-DEMOGRÁFICO E SATISFAÇÃO COM A VIDA DE IDOSOS DO MUNICÍPIO DE GUARAÍ, TOCANTINS
}

\section{DEMOGRAPHIC PROFILE AND LIFE SATISFACTION OF THE ELDERLY PEOPLE IN THE MUNICIPALITY OF GUARAÍ, TOCANTINS}

\section{PERFIL SOCIO-DEMOGRAPHIC Y SATISFACCION CON LAS VIDAS DE PERSONAS ENVEJECIDAS EN LA MUNICIPALIDAD DE GUARAÍ, TOCANTINS}

\author{
Maria Romana Friedlander ${ }^{1}$ Eliane de Fátima Gonçalves²
}

\begin{abstract}
RESUMO
Introdução: Guaraí (TO) apresenta uma porcentagem de indivíduos idosos acima da média estadual. Contudo, a literatura indica carência acentuada de estudos que descrevam as características dessa população. Objetivos: levantar os dados sociodemográficos de idosos usuários do serviço de saúde pública e verificar o grau de satisfação com a vida dessa população. Método: entrevista orientada por um formulário testado. Para a Satisfação com a vida, foi usada a Escala de Satisfação com a Vida (ESV) já publicada. Foram entrevistados 133 indivíduos. Resultados: prevalência do sexo feminino, faixa etária de 60 a 69 anos, escolaridade até 4 anos, casados, com renda mensal até 3 salários-mínimos, pardos, moradores em casa própria com até 4 coabitantes, católicos e sem vínculo empregatício. Conclusão: os dados foram relatados e demonstraram que a população está satisfeita com a vida, como ocorreu em outros estudos.
\end{abstract}

DESCRITORES: Idoso; Satisfação pessoal; Saúde Pública; Saúde do Idoso

\begin{abstract}
Introduction: Guaraí (TO) presents a percentage of elderly individuals above the state average. However, the literature indicates a remarkable lack of studies that describe the characteristics of this population. Objectives: to collect the sociodemographic data of the elderly users of the public health system and to verify the degree of life satisfaction of this population. Method: the interview guided by a form tested. For the Life Satisfaction variable, the published Life Satisfaction Scale (ESV - "Escala de Satisfação com a Vida", in Portuguese) was used. 133 individuals were interviewed. Results: female prevalence, in the age group of 60 to 69 years old, schooling up to 4 years, married, with monthly income up to 3 minimum wages, brown-skinned, residents in their own houses with up to 4 cohabitants, Catholics and without an employment relationship. Conclusion: the data are related and the results showed that the population is satisfied with their lives, as it has been verified in other studies.
\end{abstract}

KEYWORDS: Elderly; Personal satisfaction; Public health; Elderly Health

\footnotetext{
1 Enfermeira. Doutorado pela Universidade de São Paulo. Professora e Coordenadora de Pesquisa do Instituto Educacional Santa Catariana, Faculdade Guaraí (FAG), no Tocantins. E-mail: mariaromana1942@gmail.com Endereço: Rua Dr. Estevão de Almeida, 74, apart 44, CEP 05014-010. Perdizes, São Paulo (SP) - Brasil Fone: (11) 38713315.

2 Enfermeira. Ex-aluna e participante do programam de Iniciação Científica da FAG.
} 


\section{RESUMEN}

Introducción: Guaraí (TO) presenta un porcentaje de individuos ancianos por arriba de la media del estado. Sin embargo, la literatura indica marcada ausencia de estudios que describan las características de esta populatión. Objetivos: recoger los datos sociodemográficos de ancianos usuarios del servicio de salud publica y comprobar el nivel de satisfacción con la vida de esta población. Método: entrevista guiada a través de un formulario comprobado. Para la variable "Satisfacción com la vida" se utilizó la Escala de Satisfacción com la vida (ESV), ya publicada. Se entrevistaron a 133 indivíduos. Resultados: predomínio del sexo femenin, entre 60 e 69 años de edad, com uma escolaridad de hasta 4 años, casados, com un ingreso mensual de hasta 3 salarios mínimos, mestizos, habitantes com casa propia com hasta 4 co-habitantes, católicos y sin vínculo laboral. Conclusión: los datos fueron recogidos y la población está satisfecha com su vida, como lo demuestron outros trabajos de investigación.

DESCRIPTORES: Anciano; Satisfacción personal; Salud Pública; Salud del anciano 


\section{INTRODUÇÃO}

A Sociedade Brasileira de Geriatria e Gerontologia (SBGG) divulgou uma Carta Aberta à população descrevendo o contexto do envelhecimento dos idosos no Brasil e aponta algumas carências profundas em relação à saúde desse grupo. Afirma, ainda, que o enveIhecimento rápido da população, entre os brasileiros, iniciou-se em 1960 e apresenta um índice atual de 10,8\% que deve triplicar nos próximos 20 anos.

Em relação ao Estado do Tocantins, o Instituto Brasileiro de Geografia e Estatística (IBGE) ${ }^{2}$ mostra que o estado contava, em 2017, com 6,09\% de indivíduos com mais de 65 anos e projeção de $9,55 \%$ para 2030 , enquanto o país apresenta uma taxa igual a $8,46 \%$. Provavelmente, o grande contingente de indivíduos jovens que vieram trabalhar e morar no estado, após a sua fundação em 1988, explica essa taxa menor. Como nas demais regiões do país, a taxa de natalidade tocantinense está decaindo, em 2000 era de 26,08 e passou a 16,99 em 2015. Finalmente, a expectativa de vida ao nascer é igual a 71,88 anos para os homens e 79,05 para as mulheres. $O$ crescimento populacional rápido do estado, a redução da taxa de natalidade e o aumento da expectativa de vida da população tocantinense indicam um crescimento acelerado do índice de envelhecimento que, por ora, é inferior à taxa média brasileira.

Em Guaraí, o IBGE² informa que, em 2010, havia um total de 23.200 habitantes (a população estimada para 2017 era de 25.642 ) e $9,16 \%$ de idosos, o que representaria em 2017, 2349 pessoas com 60 anos ou mais. Portanto, nota-se que Guaraí apresenta tendências similares às demais regiões do país, uma vez que apresenta uma porcentagem de idosos acima da média estadual e similar à do Brasil de maneira geral.

Uma revisão da literatura brasileira nos últimos anos, aponta a existência de dados sobre o perfil sociodemográfico do indivíduo idoso em várias regiões do país, Paraná ${ }^{3}$, Paraíba ${ }^{4}$ e Rio Grande do Sul ${ }^{5}$.

A investigação do Paraná foi realizada com 359 idosos usuários de Unidades Básicas de Saúde do município de Guarapuava e informa que o grupo, em sua maioria era do sexo feminino, variando a idade entre 60 e 98 anos, média de 68,8 anos, ativos no mercado de trabalho, recebendo um salário-mínimo e casados ou morando com parceiros e em residências urbanas e multigeracionais. Encontraram ainda, 21,4\% de analfabetos. Esses autores afirmam que "os resultados obtidos podem oferecer novos subsídios para a formação dos profissionais de saúde com vistas à avaliação de saúde ampliada"3. Conclui, finalmente que os participantes, em sua maioria, estavam satisfeitos com sua vida.

O estudo realizado em João Pessoa ${ }^{4}$ utilizou uma população de 401 idosos usuários em uma Unidade de Saúde da Família apresentou predominância das muIheres, na faixa etária de 60 a 69 anos, casados, coabitando, no máximo com cinco pessoas, não sabendo ler nem escrever e com renda total do domicílio entre dois e três salários mínimos. Os autores destacam que, "apesar do envelhecimento estar associado a doenças crônicas e às incapacidades, esta associação não é imutável" e alegam que o conhecimento da população pode contribuir para a formulação e a sistematização de políticas de saúde porque "possibilitam a identificação das necessidades do grupo populacional."(Dias, et al. 2012).

Mais recentemente, uma pesquisa no Rio Grande do Sul ${ }^{5}$ realizada com 424 idosos não acamados, residentes em um município com 33.846 habitantes e $11,93 \%$ de idosos. Os resultados encontrados mostram que a maioria dos participantes era do sexo feminino, com idade média de 70,83 anos, inativos do mercado de trabalho, casados, com escolaridade inferior a 5 anos e renda familiar de 1 a 2 salários-mínimos. Os autores concluem que o conhecimento do perfil sociodemográfico pode estimular a implantação de iniciativas específicas para essa faixa etária, não só para os profissionais da área assistencial como para os gestores.

Em 2017, um estudo realizado em Maringá ${ }^{~}$ levantou as características sociodemográficas de 58 idosos com idade acima de 80 anos e verificou que se tratava de uma maioria de mulheres, viúvos, na faixa de 80-89 anos, com escolaridade básica e aposentados. Como nos demais estudos, os autores destacam a importância do conhecimento das características desta faixa etária tendo em vista o seu progressivo crescimento populacional.

Recentemente, um estudo de associação de variáveis, realizado no Minho, em Portugal ${ }^{7}$ demonstrou a relação entre alguns dados sociodemográficos e a qualidade de vida de 126 idosos e alerta os profissionais assistenciais de instituições próprias para idosos, sobre a importância do conhecimento sobre as condições de vida dos clientes para poderem canalizar seus esforços na promoção da saúde desse grupo de usuários justificando o conhecimento dos dados sociodemográficos para a melhoria da promoção da saúde dos mesmos.

No que concerne ao conhecimento das características desse contingente populacional tocantinense há uma dificuldade acentuada de bibliografia que descreva 
quem é e como é o idoso do Tocantins. Nos últimos anos foram encontrados poucos trabalhos que nos oferecem algumas informações ${ }^{8,9}$. Essa carência dificulta qualquer adequação de políticas públicas ou privadas na área da saúde, bem como impede o aparecimento de iniciativas assistenciais construídas especificamente para o idoso, o que para alguns especialistas ${ }^{3}$, trata-se de um grande desafio para o poder público, a criação e implementação de políticas para atender as novas demandas do processo do envelhecimento populacional.

Portanto, a descrição dos dados sociodemográficos permitirá, não só, a adequação e o aperfeiçoamento de iniciativas com vistas a atender as necessidades desse segmento da população, como também permitirá a construção de parâmetros para subsidiar comparações, tanto da evolução cronológica, como entre as diversas regiões do mundo e do país, como demonstraram os autores do estudo comparativo realizado em Florianópolis $^{10}$. Na conclusão do estudo realizado no Paraná os autores afirmam que o conhecimento das variáveis mencionadas pode "auxiliar os gestores a formularem indicadores de saúde"

Essas informações são indispensáveis e fundamentais para o planejamento de iniciativas assistenciais, quer sejam públicas, quer sejam privadas.

Da mesma forma, o estudo da variável "satisfação com a vida" poderá contribuir com a promoção da saúde do idoso, objeto da Atenção Básica à Saúde, uma vez que se sabe haver uma relação acentuada entre esse objeto e a variável em questão ${ }^{11}$. O trabalho realizado em Portugal ${ }^{7}$ alerta para o fato de existir uma associação negativa estreita entre o envelhecimento e a qualidade de vida e argumentam a favor da necessidade da equipe de saúde conhecer essa variável.

Um estudo realizado num município do interior do Estado de São Paulo define satisfação com a vida como "um julgamento cognitivo de alguns domínios na vida como saúde, trabalho, condições de moradia, relações sociais, autonomia entre outros, ou seja, um processo de juízo e avaliação geral da própria vida de acordo com um critério próprio"12. O mesmo estudo ainda confirmou a relação entre satisfação com a vida e algumas condições sociodemográficas, aquelas que estão relacionadas ao "bem-estar".

A carência de estudos sobre o idoso do Tocantins, especificamente em Guaraí, e suas características, justifica, não só um delineamento do perfil do idoso tocantinense, como um estudo sobre a satisfação com a vida se se considerar a relação entre a saúde e a qualidade de vida e bem-estar dessa faixa populacional tão vulnerável.

Em síntese, o problema do presente estudo centra-se na inexistência de conhecimento sobre o idoso de Guaraí o que ocasiona dificuldades para desenvolver políticas públicas e privadas adequadas e, consequentemente leva à falta de qualidade de vida e bem-estar e carência de parâmetros para avaliar a evolução e fazer comparações com outros grupos populacionais.

Portanto, espera-se que os resultados obtidos possibilitem identificar necessidades específicas, estimulem a implantação de iniciativas adequadas, melhorem a promoção da saúde, possibilitem a adequação das políticas, permitam a construção de parâmetros para estudar a evolução e estabelecer comparações e auxiliem a elaborar metas e indicadores de atuação.

Considerando a importância desse conhecimento e a relação entre a saúde e a satisfação com a vida, o presente estudo tem como proposição: levantar os dados sócio demográficos e o grau de satisfação com a vida dos idosos usuários das Unidades de Atenção Básica e do Centro de Convivência de Guaraí (TO), ou seja, dos recursos que o município oferece para a saúde dos idosos.

\section{MÉTODO}

Trata-se de uma pesquisa descritiva com coleta de dados transversal e abordagem quantitativa realizada no município de Guaraí (Tocantins).

A população foi constituída por 133 idosos, com 60 anos ou mais, o que significa uma amostra de 5,66\% dos indivíduos idosos, estimados pelo IBGE, para 2017, no município de Guaraí.

Os critérios de inclusão foram, além de ter 60 anos ou mais, estar cadastrado nas unidades básicas de saúde (UBS) ou Centro Municipal de Convivência do município de Guaraí (TO), presentes na unidade no horário visitado pela entrevistadora e que aceitassem responder às perguntas do formulário. Portanto, não se trata de uma amostra probabilística, mas foi absolutamente não intencional. Note-se que se trata de uma população de usuários do Sistema Único de Saúde, com necessidades assistenciais.

As entrevistas foram efetivadas, após autorização administrativa dos órgãos municipais, por uma das autoras, moradora local e, portanto, conhecedora dos costumes, vocabulário e forma de iniciar a interação, o que facilitou a comunicação e conquistou rapidamente a confiança dos idosos. $O$ fato de ter apenas um entrevistador impediu vieses e falhas de sistematização ocasionadas 
por estilos pessoais variados na entrevista. Além disso, dificultou a violação do anonimato e o uso inadequado dos dados coletados, diminuindo possíveis riscos.

A entrevistadora convidou cada um dos participantes possíveis para a entrevista que foi realizada na própria UBS; todos os participantes assinaram o Termo de Consentimento Livre e Esclarecido (TCLE) após as explicações sobre a importância do estudo, os objetivos do mesmo e os direitos dos entrevistados. Acredita-se que as condições mentais dos idosos entrevistados permitiram a compreensão do texto do TCLE e as condições de sua concordância porque essa dinâmica foi testada durante as iniciativas do plano piloto. Não houve, nesse sentido nenhuma intercorrência desagradável e, foram raros os casos de não aceitação da entrevista que demorou em média, 10 minutos.

Utilizou-se um formulário que demonstrou, no pré-teste, ser confiável em relação aos dados colhidos. As variáveis socio demográficas foram fundamentadas em trabalhos anteriores ${ }^{13-16}$ e são as seguintes: sexo, idade, estado conjugal, religião, escolaridade, ocupação com vínculo empregatício, renda mensal familiar, moradia, número de moradores e cor auto declarada. Note-se que as características da população de estudo podem ser observadas no Quadro 1 dos resultados.

Para a variável Satisfação com a vida utilizou-se a Escala de Satisfação com a Vida (ESV) já validada em estudos anteriores nacionais ${ }^{17-19}$ e internacionais ${ }^{16}$, em adultos $^{17}$ e idosos de baixa escolaridade ${ }^{13}$. O método para a aplicação dessa escala foi aquele descrito em $2010^{18}$, ou seja, entrevistas conduzidas por um formulário.

O projeto da pesquisa foi submetido à apreciação do Comitê de Pesquisa do Centro Universitário Luterano de Palmas e recebeu o número do CAAE 90497517.8.0000.5516. Foi aprovado em 07 de junho de 2018.
Os dados obtidos foram analisados com a utilização das medidas de tendência central. A associação entre os grupos, ou seja, a verificação de resultados significativos entre a pontuação da Satisfação com a Vida e as variáveis sociodemográficas foi estudada por meio da aplicação do teste Qui-quadrado $\left(\mathrm{X}^{2}\right)$ ao nível de significância de 5\% e nível de confiança de 95\%, com a correção pelo Coeficiente de Yates nos casos em que o resultado da aplicação do teste de Qui-quadrado deu significativo e as tabelas apresentavam números abaixo de 5 (variáveis "cor autodeclarada" e "moradia").

\section{RESULTADOS}

Os resultados estão apresentados na seguinte ordem: características sócio demográficas dos idosos entrevistados, pontuação da ESV e as possíveis associações entre as variáveis sociodemográficas e a pontuação obtida na ESV.

As características sociodemográficas da população do estudo podem ser observadas na Tabela 1.

Observou-se que as frequências absolutas e porcentuais da população que se destacam são: o número de participantes do sexo feminino é levemente superior ao do masculino, a faixa de idade dos 60-69 é levemente superior à de 70-79, a escolaridade da grande maioria dos entrevistados é de 1 a 4 anos de estudo, a maior parte é casada mas o grupo de solteiros, viúvos e divorciados ou separados é a maioria $(51,12 \%)$, a renda mensal da quase totalidade de idosos é de até 3 salários-mínimos; a cor autodeclarada com maior frequência é a parda, a grande maioria dos idosos mora em casa própria com até 4 moradores, a maior parte adotou a religião católica e não têm ocupação com vínculo empregatício. Note-se, ainda, que se destaca os $9,78 \%$ de idosos com 9 ou mais anos de escolaridade. 
Tabela 1 - Características sócio demográficas dos idosos. Guaraí (TO). 2018

\begin{tabular}{|c|c|c|}
\hline Características populacionais & $\mathrm{N}^{0}$ & $\%$ \\
\hline $\begin{array}{l}\text { SEXO } \\
\text { Masculino } \\
\text { Feminino } \\
\text { Total }\end{array}$ & $\begin{array}{l}64 \\
69 \\
133\end{array}$ & $\begin{array}{l}48,12 \\
51,88 \\
100\end{array}$ \\
\hline $\begin{array}{l}\text { IDADE ( } n^{\circ} \text { de anos) } \\
60-69 \\
70-79 \\
80 \text { ou mais } \\
\text { Total }\end{array}$ & $\begin{array}{l}62 \\
55 \\
16 \\
133\end{array}$ & $\begin{array}{l}46,63 \\
41,35 \\
12,02 \\
100\end{array}$ \\
\hline $\begin{array}{l}\text { ESCOLARIDADE }\left(n^{\circ} \text { de anos }\right) \\
1-4 \\
5-8 \\
9 \text { ou mais } \\
\text { Total }\end{array}$ & $\begin{array}{l}113 \\
07 \\
13 \\
133\end{array}$ & $\begin{array}{l}84,97 \\
5,25 \\
9,78 \\
100\end{array}$ \\
\hline $\begin{array}{l}\text { ESTADO CONJUGAL* } \\
\text { Solteiro } \\
\text { Casado/morando junto } \\
\text { Viúvo } \\
\text { Divorciado/separado } \\
\text { Total }\end{array}$ & $\begin{array}{l}10 \\
65 \\
42 \\
16 \\
133\end{array}$ & $\begin{array}{l}7,52 \\
48,88 \\
31.58 \\
12.02 \\
100\end{array}$ \\
\hline $\begin{array}{l}\text { RENDA FAMILIAR (em salários } \\
\text { mínimos) } \\
\text { Até } 3 \\
4-6 \\
\text { Mais de } 6 \\
\text { Total }\end{array}$ & $\begin{array}{l}130 \\
3 \\
- \\
133\end{array}$ & $\begin{array}{l}97,75 \\
2,25 \\
- \\
100\end{array}$ \\
\hline $\begin{array}{l}\text { COR AUTODECLARADA } \\
\text { Preta } \\
\text { Parda } \\
\text { Branca } \\
\text { Total }\end{array}$ & $\begin{array}{l}4 \\
118 \\
11 \\
133\end{array}$ & $\begin{array}{l}3,00 \\
88,73 \\
8,27 \\
100\end{array}$ \\
\hline $\begin{array}{l}\text { MORADIA } \\
\text { Própria } \\
\text { Alugada } \\
\text { Cedida } \\
\text { Outros (Qual?) }\end{array}$ & $\begin{array}{l}116 \\
12 \\
05 \\
133\end{array}$ & $\begin{array}{l}87,22 \\
9,02 \\
3,76 \\
100\end{array}$ \\
\hline $\begin{array}{l}\text { NÚMERO DE MORADORES } \\
\text { Até } 2 \\
3-4 \\
5-6 \\
\text { Mais de } 6 \\
\text { Total }\end{array}$ & $\begin{array}{l}47 \\
68 \\
11 \\
07 \\
133\end{array}$ & $\begin{array}{l}35,33 \\
51,12 \\
8,29 \\
5,26 \\
100\end{array}$ \\
\hline $\begin{array}{l}\text { RELIGIÃO } \\
\text { Sem religião } \\
\text { Com religião/não praticante (Qual?) } \\
\text { Praticante: Católica } \\
\quad \quad \text { Evangélica } \\
\quad \text { Outras (Qual?) } \\
\text { Total }\end{array}$ & $\begin{array}{l}12 \\
09 \\
83 \\
28 \\
01 \\
133\end{array}$ & $\begin{array}{l}9,02 \\
6,76 \\
62,41 \\
21,06 \\
0,75 \\
100\end{array}$ \\
\hline $\begin{array}{l}\text { OCUPAÇÃO } \\
\text { Não } \\
\text { Sim (Qual?) } \\
\text { Total }\end{array}$ & $\begin{array}{l}105 \\
28 \\
133\end{array}$ & $\begin{array}{l}78,95 \\
21,05 \\
100\end{array}$ \\
\hline
\end{tabular}

* Nota: os participantes que vivem sem companheiro (solteiros, viúvos, divorciados ou separados) totalizam 68 ou $51,12 \%$.

A Tabela 2 apresenta os resultados relacionados à Escala de Satisfação com a Vida (ESV). Apesar da maioria dos idosos declarar que sua satisfação com a vida estar abaixo da média, verifica-se que essa média está alta, ou seja, supera os 17,5 que são a metade da maior pontuação possível.

Tabela 2 - Pontuação do teste de Satisfação com a vida declarada pelos idosos de Guaraí (TO). 2018

\begin{tabular}{|l|l|l|}
\hline PONTUAÇÃO & $\mathrm{N}^{\circ}$ & $\%$ \\
\hline Igual ou Acima da média & 64 & 48,12 \\
\hline Abaixo de média & 69 & $\mathbf{5 1 , 8 8}$ \\
\hline Total & 133 & 100 \\
\hline Média obtida = 27 pontos & \\
\hline Pontuação máxima obtida $=35$ & \\
\hline Pontuação mínima obtida $=16$
\end{tabular}

Na Tabela 3 podem ser observadas as associações entre as variáveis sociodemográficas e a pontuação na Escala de Satisfação com a Vida. Nessa tabela foi indicada a média de pontos obtida pela população de estudo. Não foi encontrada nenhuma associação entre os grupos analisados mas o número de moradores por residência em associação com a satisfação com a vida, deu um resultado limítrofe $\left(x^{2}=5,98\right)$, ou seja, novas pesquisas devem levantar hipóteses coerentes com a possibilidade de muitos moradores na residência do idoso influenciar negativamente seu bem estar. 
Tabela 3 - Relação da Satisfação com a vida e as características sóciodemográficas. Guaraí (TO). 2018.

\begin{tabular}{|c|c|c|c|c|c|}
\hline \multirow{2}{*}{ SATISFAÇÃO COM VIDA CARACT. } & \multicolumn{2}{|c|}{ Igual ou Acima da Média } & \multicolumn{2}{|c|}{ Abaixo da média } & \multirow[t]{2}{*}{ Teste de significância* } \\
\hline & $\mathrm{N}^{\circ}$ & $\%$ & $\mathrm{~N}^{\circ}$ & $\%$ & \\
\hline SEXO & & & & & $x^{2} \mathrm{cal}<3,84$ \\
\hline Masculino & 35 & 54,69 & 29 & 42,03 & \\
\hline Feminino & 29 & 45,31 & 40 & 57,97 & \\
\hline Total & 64 & 100 & 69 & 100 & \\
\hline IDADE (em anos) & & & & & $\mathrm{X}^{2} \mathrm{cal}<5,99(=5,58)$ \\
\hline $60-69$ & 24 & 36,92 & 38 & 55,87 & \\
\hline $70-79$ & 29 & 44,61 & 26 & 38,23 & \\
\hline 80 ou mais & 11 & 17,19 & 05 & 7,24 & \\
\hline Total & 64 & 100 & 69 & 100 & \\
\hline ESCOLARIDADE ** (em anos) & & & & & $x^{2} \mathrm{cal}<3,84$ \\
\hline $1-4$ & 52 & 81,25 & 61 & 88,40 & \\
\hline 5 ou mais & 12 & 18,75 & 08 & 11,60 & \\
\hline Total & 64 & 100 & 69 & 100 & \\
\hline ESTADO CONJUGAL ** & & & & & $\mathrm{x}^{2} \mathrm{calc}<3,84$ \\
\hline Solteiro, viúvo, divorciado ou separado & 34 & 53,13 & 34 & 49,27 & \\
\hline Casado/morando junto & 30 & 46,87 & 35 & 50,72 & \\
\hline Total & 64 & 100 & 69 & 100 & \\
\hline RENDA FAMILIAR ** (Salários Mínimos) & & & & & $x^{2} \mathrm{cal}<3,84$ \\
\hline Até 3 & 61 & 96,82 & 68 & 98,55 & \\
\hline Mais de 3 & 03 & 4,68 & 01 & 1,42 & \\
\hline Total & 64 & 100 & 69 & 100 & \\
\hline COR AUTODECLARADA ** & & & & & $\mathrm{x}^{2} \mathrm{cal}>3,84$ Yates $^{\star * *}$ \\
\hline Parda & 61 & 93,31 & 57 & 88,40 & \\
\hline Branca/Preta & 03 & 4,69 & 12 & 11,60 & \\
\hline Total & 64 & 100 & 69 & 100 & \\
\hline MORADIA ** & & & & & $x^{2}$ cal $>3,84$ Yates ${ }^{\star \star *}$ \\
\hline Própria & 60 & 93,75 & 56 & 81,16 & \\
\hline Alugada/Cedida & 04 & 6,24 & 13 & 18,84 & \\
\hline Total & 64 & 100 & 69 & 100 & \\
\hline $\mathrm{N}^{\circ}$ DE MORADORES ** & & & & & $X^{2} \mathrm{cal}<5,99(=5,98)$ \\
\hline Até 2 & 27 & 42,19 & 20 & 28,99 & \\
\hline $3-4$ & 28 & 43,75 & 40 & 57,97 & \\
\hline 5 ou mais & 09 & 14,06 & 09 & 13,04 & \\
\hline Total & 64 & 100 & 69 & 100 & \\
\hline RELIGIÃO ** & & & & & $x^{2}$ calc $<3,84$ \\
\hline Sem religião/ não praticante & 07 & 10,94 & 14 & 20,29 & \\
\hline Praticante & 57 & 89,06 & 55 & 79,71 & \\
\hline Total & 64 & 100 & 69 & 100 & \\
\hline OCUPAÇÃO & & & & & $x^{2}$ calc $<3,84$ \\
\hline Sim & 16 & 25,00 & 12 & 17,39 & \\
\hline Não & 48 & 75,00 & 57 & 82,61 & \\
\hline Total & 64 & 100 & 69 & 100 & \\
\hline
\end{tabular}

* Não há associação entre os grupos analisados.

** Foram agrupadas algumas opções para permitir o uso do teste do Qui-quadrado $\left(x^{2}\right)$

*** Com a correção de Yates, $x^{2}<3,84$ 


\section{DISCUSSÃO}

Como já foi mencionado, trata-se de uma população em atendimento na Unidade Básica de Saúde e, portanto, com necessidades assistenciais. Este fato, deve ser considerado na interpretação dos resultados do presente trabalho.

Em relação ao sexo, que excepcionalmente no Tocantins o masculino é predominante, encontrou-se uma maioria do sexo feminino, fato também encontrado em trabalho anterior, realizado em Guarai ${ }^{20}$ e explicado por vários autores como consequência da mulher ter mais disponibilidade de tempo, valorizar a manutenção da saúde da família, aderir mais facilmente aos cuidados médicos e pelos comportamentos negativos dos homens relacionados à dificuldade para reconhecer suas fragilidades ${ }^{20}$.

Quanto à idade, como se espera, a faixa dos idosos mais novos (60-69) caracterizou a maior parte da população de estudo $(46,63 \%)$. Esse fato, pela sua coerência pode argumentar a favor da confiabilidade interna do método de coleta do presente estudo.

Os resultados relacionados à escolaridade demonstram que os usuários idosos das UBS, em maioria absoluta, frequentaram a escola de 1 a 4 anos o que se considera uma escolaridade baixa, porém semelhante a outros trabalhos com populações similares ${ }^{8,13,21}$. Contudo, surpreende terem sido encontrados $9,78 \%$ idosos com 9 ou mais anos de escola. Esse resultado é similar ao encontrado em trabalho anterior realizado em Goiânia com população similar ${ }^{13}$. Uma hipótese a ser levantada é que o melhor nível de escolaridade associada a renda mais alta permite maior acesso ao conhecimento sobre saúde e proporciona oportunidades de fazer uso de serviços de saúde de maior qualidade.

Em relação ao estado conjugal, verifica-se que uma grande parte da população estudada é de casados ou coabitando com parceiros. Entretanto, os solteiros, viúvos, separados ou divorciados, ou seja, sem parceiro, totalizam $51,30 \%$, a maioria. Este fato difere do encontrado por outros autores que observaram a maioria de casados $4,5,10,13,15$.

Os resultados relacionados à renda familiar (até 3 salários mínimos 97,75\%), cor autodeclarada (parda $88,73 \%$ ), moradia (própria 87,22 ), religião (católica $62,41 \%$ ) e ocupação (sem vínculo empregatício $78,95 \%$ ) coincidem, aproximadamente, com os encontrados em outras pesquisas $3,4,9,10,13,15,16$.
Em relação ao segundo objetivo, ou seja, levantar a pontuação da satisfação com a vida desta população, percebe-se que a média da pontuação é bastante alta (27) se a compararmos com a média possível, ou seja, a metade da máxima pontuação possível $(17,5)$. Este fato indica uma alta satisfação com a vida dos idosos pesquisados. Apesar de estarem com necessidades de saúde a serem atendidas, os idosos, usuários do SUS de Guaraí percebem-se como pessoas com bom nível de bem-estar. Esse resultado coincide com os resultados de investigação nacional ${ }^{12}$. Mesmo aquela porcentagem populacional que ofereceu respostas à ESV abaixo da média, tendo em vista que a média é alta, ainda demonstraram que estão satisfeitos com sua vida. Encontrou-se apenas 7 elementos que pontuaram a ESV abaixo de 17,5, ou seja, percebiam-se insatisfeitos com a vida. Achou-se interessante verificar se alguma das variáveis sociodemográficas estaria associada à pontuação da ESV. Os resultados analisados (Quadro 3) mostram que, com exceção da idade e do número de moradores, não há associação entre as variáveis sociodemográficas e a satisfação com a vida.

A idade e o número de moradores na residência e sua associação com a pontuação da ESV apresentaram valores do qui-quadrado limítrofes o que indica a necessidade de novas pesquisas específicas.

\section{CONCLUSÃO}

As informações obtidas neste estudo mostram que os objetivos delineados foram atendidos.

No que concerne ao perfil sociodemográfico, conclui-se que a população de idosos, usuários dos recursos municipais de saúde de Guaraí, possui as seguintes características: a) prevalência de indivíduos do sexo feminino; b) a maior parte da população idosa está na faixa etária de 60 a 69 anos; c) a escolaridade é muito baixa; d) é casada; e) é de cor parda; f) vive com a renda mensal de até 3 salários-mínimos; g) mora em casa própria; h) coabita com até 4 moradores; i) é católica; e j) não tem ocupação com vínculo empregatício. Chama a atenção o fato de $9,78 \%$ dos idosos terem 9 ou mais anos de escolaridade. Os destaques citados são similares aos de outros trabalhos brasileiros.

Quanto à variável "Satisfação com a vida", os resultados foram coerentes com outros trabalhos e mostraram que a população do estudo se sente satisfeita com sua a vida. 
Não foi encontrada nenhuma variável sociodemográfica associada ao nível de satisfação com a vida. Contudo, sugere-se que novas pesquisas sejam efetuadas para verificar a associação entre o nível de satisfação com a vida e as variáveis "idade" e "número de moradores na residência".
Finalmente, na interpretação destes resultados é necessário considerar que a população, quando informou seus dados e opiniões, estava em situação de atendimento de saúde, portanto, com algum problema que hipoteticamente poderia interferir na sua percepção de bem-estar. 


\section{REFERÊNCIAS}

1 - Sociedade Brasileira de Gerontologia e Geriatria (SBGG). Envelhecimento no Brasil e Saúde do Idosos: Carta Aberta à população 2014. [acesso em 23 mar 2016]. Disponível em: http://.www.sbgg.org.br/c/artigos/.

2 - Instituto Brasileiro de Geografia e Estatística (Brasil). Projeção da População do Brasil e das Unidades Federativas, Tocantins, Guaraí 2017. [acesso em: 06 abril 2018]. Disponível em: http://www.ibge.gov.br/apps/ populacao/projecao.

3 - Pilger C, Menon MH, Mathias TAF. Características sociodemográficas e de saúde de idosos: contribuições para os serviços de saúde. Rev. Latino-Am. Enfermagem 2011;19(5):1230-8.

4 - Dias LD, Brito GEG, Forte FDS, Araújo KMB, Lucena EMF. Perfil sociodemográfico e de Saúde de Idosos do Município de João Pessoa-PB. Rev. Bras. Promoç. Saúde. 2012;25(1):86-96.

5 - Luz EP, Dollepione LB, Kirchener RM, Silva LAA, Silva FP, Kohler J, Gopinter E, Carlot JM. Perfil sociodemográfico e de hábitos de vida da população idosa de um município da região norte do Rio Grande do Sul, Brasil. Rev. Bras. Geriatr. Geronto 2014;17(2):303-14.

6 - Jorge MSG, Lima WG, Vieira PR, Vogelmann SC, Myra RS, Wibelinger LM. Caracterização do Perfil sociodemográfico, das condições de Saúde e das condições sociais de idosos octogenários. Saúde e Pesquisa 2017;10(1):61-73.

7 - Roncon J, Lima S, Pereira MG. Qualidade de vida, Morbilidade Psicológica e Stress Familiar em Idosos residentes na Comunidade. Psicologia: Teoria e Pesquisa 2015;31(1):87-96.

8 - Aguiar MA. Idoso institucionalizado e a caracterização das instituições de longa permanência no Estado do Tocantins. Dissertação de Mestrado em Gerontologia. Brasília DF: Universidade Católica de Brasília; 2013.
9 - Pereira LC, Prestes J, Melo GF, Silva Neto LS, Funghetto SS, Pires AB, Boff G, Alves AT, Karnikowski MGO. A influência da composição corporal na força de homens idosos brasileiros. Rev Bras Med Esporte. 2015;21(3):187-199.

10 - Antes DL, Ribeiro DF, Schnaider IJC, Bennedeti TRB, D'Orsi E. Perfil socioeconômico dos idosos de Florianópolis: análise comparativa dos estudos Perfil do Idoso 2002 e EpiFloripa Idoso 2009. Rev. Bras. Epidemiol. 2014;17(1):189-202.

11 - Scorsolini-Comin F, Santos MA. O estudo científico da felicidade e a promoção da saúde: revisão integrativa da literatura. Rev. Latino-Am. Enferm. 2010;18(3):472-9.

12 - Joia LC, Ruiz T, Donalisio MR. Condições associadas ao grau de satisfação com a vida entre a população de idosos. Rev. Saúde Pública. 2007;41(1):131-138.

13 - Nunes DP, Nakatani AYK., Silveira EA, Bachion MM, Souza, MR. Capacidade funcional, condições socioeconômicas e de saúde de idosos atendidos por equipes de Saúde da Família de Goiânia (GO, Brasil). Ciência e Saúde Coletiva 2010;15(6): 2887-2898.

14 - Moreira S, Ferreira TR, Borges E. Qualidade de vida e satisfação com a vida dos idosos na comunidade. In. III Congresso Internacional da Sociedade Portuguesa de Enfermagem em Saúde Mental (SPESM); 2012; Porto (Portugal), E-book: 643-655.

15 - Porciúncula RCR, Carvalho EF, Barreto KML, Leite, VMM. Perfil sócio epidemiológico e autonomia de longevos em Recife-PE, Nordeste do Brasil. Rev. Bras. Geriatr. Geronto. 2014;17(2):315-325.

16 - Oliveira BLCA, Silva AM da, Silva RA da, Thomaz EBF. Racial inequalities in the socioeconomic, demographic and health conditions of eldery from Maranhão State, Legal Amazon, Brazil: a population-based study. Acta Amazônica 2014;44(3):335-344. 
17 - Gouveia VV, Barbosa GA, Andrade EO, Carneiro MB. Medindo a satisfação com a vida dos médicos do Brasil. J Bras Psiquiatr. 2005;54(4):298-305.

18 - Albuquerque FJB, Sousa FM, Martins CR. Validação das escalas de Satisfação com a Vida e afetos para idosos rurais. Psico Porto Alegre PUCRS 2010;41(1):85-92.

19 - Carneiro RS, Falcone EMO. O desenvolvimento das habilidades sociais em idosos e sua relação na satisfação com a vida. Estudos de Psicologia 2013;18(3):517526.
20 - Friedlander MR, Guimarães CRR, Fabichacki E. O perfil do usuário de uma unidade básica de saúde integrada a uma faculdade privada. Desafios 2016;3(2):03-13.

21 - Guths JFS, Jacob MHVM, Santos AMPV, Arossi GA, Béria JU. Socidemographic profile, family aspects, perception of health, functional capacity and depression in institutionalized eldery persons from the north coastal region of Rio Grande do Sul, Brazil. Rev. bras. geriatr. gerontol. 2017;20(2):175-185. 